\title{
Mid-morning Tryptophan Depletion Delays REM Sleep Onset in Healthy Subjects
}

\author{
Isabelle Arnulf, M.D., Philippe Quintin, M.D., Jean-Claude Alvarez, Ph.D., Laurence Vigil, \\ Yvan Touitou, Ph.D., Anne-Sophie Lèbre, Ph.D., Agnès Bellenger, Odile Varoquaux, Ph.D., \\ Jean-Philippe Derenne, M.D., Jean-François Allilaire, M.D., Chawki Benkelfat, M.D., \\ and Marion Leboyer, M.D., Ph.D.
}

\begin{abstract}
Because serotonin is involved in the diachronic regulation of sleep, we tested the effect of a midmorning rapid deficiency in the serotonin precursor tryptophan on the next night's sleep. After a 48-h low-protein diet, 17 healthy volunteers received either a tryptophan-free mixture of amino acids or a placebo at 10:30 A.M., in a randomized double-blind cross-over design, resulting in a $77 \%$ decrease and $41 \%$ decrease of serum tryptophan at 3:30 P.M. and 9:30 P.M., respectively. Urinary sulfatoxy-melatonin excretion and mood were unaffected by the rapid tryptophan depletion (RTD), but rapid eye movement (REM) sleep
\end{abstract}

latency increased by $21 \mathrm{~min}$ (from $91.5 \pm 4.5 \mathrm{~min}$ to $112.2 \pm$ $6.9 \mathrm{~min}$ ), sleep fragmentation $58 \%$, and REM density of the first REM sleep period doubled. The results show that midmorning RTD delays REM sleep latency during following night-time sleep, whereas evening RTD shortens REM sleep latency in previous studies, and suggest that the serotonin control of REM sleep latency is upregulated.

[Neuropsychopharmacology 27:843-851, 2002] (C) 2002 American College of Neuropsychopharmacology. Published by Elsevier Science Inc.

\section{KEY WORDS: Serotonin; Tryptophan; Sleep; REM sleep}

The importance of serotonin in the regulation of sleep has been demonstrated by anatomic, electrophysiological, and pharmacological studies in animal models. In

From AP-HP, Centre d'Investigation Clinique, Fédération des Pathologies du Sommeil et UPRES EA 2397 (IA, LV, A-SL, J-PD), Service de Biochimie Médicale (YT), Pharmacie (AB), Service de Psychiatrie (PQ, JFA), Hôpital Pitié-Salpêtrière, Paris, Assistance Publique-Hôpitaux de Paris, Service de Psychiatrie, Hôpital Albert Chenevier and Henri Mondor, Créteil (ML); Biochimie, Hôpital Mignot, Le Chesnay (J-CA), France, and Department of Psychiatry, McGill University, Montréal, Canada (CB).

Address correspondence to: Dr. Isabelle Arnulf, Fédération des Pathologies du Sommeil, Hôpital Pitié-Salpetriere, 47-83 Boulevard de l'Hôpital, 75651 Paris Cedex 13, France. Tel.: 33 (1)- 42-17-67-51; Fax: 33 (1)- 42-17-68-43; E-mail: isabelle.arnulf@psl.ap-hop-paris.fr

Received August 9, 2001; revised April 10, 2002; accepted April 20, 2002.

Online publication: $4 / 25 / 02$ at www.acnp.org/citations/ Npp042502293. cats the inhibition of the serotonin synthesis by parachlorophenylalanine or the destruction of serotonin-containing neurons of the raphe dorsalis (Jouvet 1972) causes total insomnia for more than 48 h. However, the release of serotonin (Cespuglio et al. 1983) and the activity of dorsal raphe nucleus serotonergic neurons (Trulson and Jacobs 1979) are at their highest during waking, decrease during non-rapid eye movement (nonREM) sleep, and are suppressed during rapid eye movement (REM) sleep. In order to reconcile these apparently conflicting results, Jouvet proposed that the release of serotonin during period of wakefulness conditions the later synthesis of sleep-inducing factors (Jouvet 1984).

On the other hand, serotonin has been shown to inhibit REM sleep, and is implicated in the pathophysiology and treatment of major depression. The synthesis of serotonin in the brain depends on plasma tryptophan levels (Fernstrom and Wurtman 1974). Serotonin synthesis in human brain can therefore be reduced (Nish- 
izawa et al. 1997) by ingestion of a tryptophan-free amino acid mixture that lowers plasma tryptophan levels by $80 \%$ and inhibits transport across the blood-brain barrier (Young et al. 1985). This rapid tryptophan depletion (RTD) is a method that temporarily and reversibly deactivates the serotonin system. Previous research has indicated that decreasing serotonergic neurotransmission via the RTD exerts changes on mood and sleep. RTD may temporarily reverse the antidepressant effects of selective serotonin re-uptake inhibitors in fully remitted depressed patients (Delgado et al. 1990). RTD depresses mood in healthy subjects at genetic risk for major depressive disorders (Benkelfat et al. 1994), and induces relapse of depression in stable drug-free women with previous suicidal tendencies (Smith et al. 1997), but not in a similar group including patients of both sex without former suicidal tendencies (Leyton et al. 1997).

Patients with major affective disorders may present REM sleep abnormalities including a shortened REM sleep latency and an increased REM density during REM sleep (Kupfer 1976), an effect reversed by drugs enhancing serotonin brain transmission (Trivedi et al. 1999). The shortened REM sleep latency is also found in healthy siblings of patients with major affective disorders (Giles et al. 1998). These results suggest that there could be a causal relationship between low brain serotonin transmission, low mood and REM sleep abnormalities.

One may thus expect RTD to either induce a total insomnia (as this is shown for severe serotonin depletion in animal models) or shorten REM sleep latency (as this is observed in patients with major affective disorder suspect of having low serotonin brain levels). In healthy subjects, RTD applied in the evening never induced a total insomnia. It decreased sleep stage 2 (Bhatti et al. 1998; Voderholzer et al. 1998) but either decreased (Bhatti et al. 1998) or unchanged REM sleep latency (Voderholzer et al. 1998). In fully remitted male patients treated with selective serotonin re-uptake inhibitors, RTD reduced sleep latency, REM sleep latency, and increased rapid eye movement density, time and percentage (Moore et al. 1998). In these studies, plasma tryptophan levels were lowest at sleep onset. However, if one considers that serotonin brain transmission is normally low during sleep, one may expect that RTD applied in the evening would have less depressing effect on brain serotonin during sleep than RTD applied during daytime wakefulness, when serotonin levels are at their highest. To date, no studies have used a tryptophan depletion challenge to examine the effect of daytime RTD on sleep. We therefore studied effects of midmorning RTD on subsequent night sleep in healthy volunteers, using a placebo-controlled randomized crossover design. We expected to obtain a marked shortening in REM sleep latency.

\section{METHODS}

\section{Subjects}

Eighteen healthy subjects, 11 women and 7 men, aged 18 to 38 years (mean: 26 years, SD: 5.9 years), with a mean body mass index of $22.2 \pm 4.3 \mathrm{~kg} / \mathrm{m}^{2}$ (range 17.633) were recruited among medical and nursing students by an advertisement. The volunteers gave informed written consent to participate to the study, which was approved by the local ethics committee. They were included in the study after being interviewed by a psychiatrist (PQ) with a standardized instrument (Diagnosis Interview for Genetic Study, DIGS) designed to rule out present or lifetime history of psychiatric disorders and family history of affective disorders in first and second degree relatives (Nurnberger et al. 1994). Of the 44 subjects interviewed, 26 were rejected because of personal axis I diagnosis and/or family history of affective disorders in first and second degree relatives. Medical health (clinical history, absence of drugs or dietary restrictions, standardized clinical examination) was checked before inclusion by a neurologist and sleep doctor (IA), as well as the absence of prior history of sleep disorders. There was no screening or adaptation night polygraphic study. The subjects did not have transmeridian flights in the month preceding the study and were compelled to follow stable sleepwake cycles (bedtime: 11 P.M. \pm 1 , wake up time: 7 P.M. \pm 1) one week before and throughout the study. One subject snored. One woman received oral metoclopramide after vomiting the amino acid drink, and was withdrawn from the sleep study. The subjects received money compensation after their participation to the study.

\section{Design}

As shown in Table 1, subjects were restricted to a lowtryptophan diet (breakfast, lunch, dinner, composed from a list of authorized food and drinks) at home two days before the administration of the aminoacid mixture or the placebo. For example, tea, coffee (no milk), bread, jam and honey were authorized at breakfast. Vegetables, potatoes, rice and fruits were authorized at lunch and diner. The consumption of meat, fish, eggs, soybean, sweets, muësli and alcohol were forbidden. The study was performed on day 3 in the clinical research center. At 7:45 A.M., venous blood samples were drawn. At 8:50 A.M., the subjects, who had fasted since midnight the previous night, were asked to void their urine. Mood was rated with the Hamilton scale version A (Hamilton 1960) by a psychiatrist (PQ) blind to the experimental condition and the Bipolar Form of the profile of mood states (POMS, an auto-questionnaire constructed to measure six bipolar subjective mood states (Lorr and McNair 1982)): I Composed-Anxious; II 
Agreeable-Hostile, III Elated-Depressed, IV ConfidentUnsure, V Energetic-Tired, VI Clearheaded-Confused) at 9:00 A.M. At 10:30 A.M., either a tryptophan-free amino acid or a placebo drink was administered in a randomized, double blind fashion with a minimum seven days between trials. Blood samples were drawn and mood ratings were performed at 3:30 P.M., after 5-h tryptophan depletion. A low-tryptophan snack was served at 5:30 P.M. (fruit juice, gluten-free toasts, glutenfree biscuits, stewed apple, fresh fruit) and 7:30 P.M. (green salad, gluten-free pasta, cooked lettuce and carrots, gluten-free toasts and biscuits, fresh fruit, fruit juice, stewed apple and fruits with syrup). Blood samples were drawn again at 9:30 P.M., after an 11-h depletion, and sleep was recorded between 11:00 P.M. and 7:30 A.M. At 9:00 A.M. on day 4, a breakfast was served in order to build up serotonin again. It included coffee with milk and sugar, yogurt, bread with butter and jam, cheese $(30 \mathrm{~g})$, ham $(30 \mathrm{~g})$, fruit juice and chocolate.

\section{Tryptophan Depletion}

The tryptophan-free amino acid mixture was similar as the one used by Young et al. (1985). Fifteen powdered amino acids (no tryptophan) including L-alanine, $5.5 \mathrm{~g}$; L-arginine, 4.9 g; L-cysteine, 2.7 g; glycine, 3.2 g; L-histidine, 3.2 g; L-isoleucine, 8 g; L-leucine, 13.5 g; L-lysine monohydrochloride, $11 \mathrm{~g}$; L-methionine, $3 \mathrm{~g}$; L-phenylalanine, $5.7 \mathrm{~g}$; L-proline, $12.2 \mathrm{~g}$; L-serine, $6.9 \mathrm{~g}$; L-threonine, $6.9 \mathrm{~g}$; L-tyrosine, $6.9 \mathrm{~g}$; and L-valine, $8.9 \mathrm{~g}$ were mixed with $2 \mathrm{~g}$ aspartame, $14 \mathrm{~g}$ cacao powder, and 5 drops of mint syrup in $250 \mathrm{ml}$ of water. Since the use of tryptophan is forbidden in France because of the risk of hypereosinophilia-myalgia syndrome (Belongia et al. 1990), the placebo mixture contained only cacao powder, mint syrup and aspartame. Methionine, arginine and cysteine were given in capsules because of their unpleasant taste. Patients receiving the placebo were given capsules containing lactose. As verified in a preliminary test, the subjects could not taste the difference between the placebo and the amino-acid mixture and could not visually tell the difference between capsules. All products were prepared just before use and were controlled by the pharmacists of the Pitié-Salpêtrière Hospital (ASL, AB), who also kept secret the randomization code.

\section{Polysomnography}

Polysomnography was performed from 11:00 P.M. (lights off) to 7:30 A.M. (lights on) as previously described (Arnulf et al. 2000). Sleep stages and arousal were scored visually according to standard criteria (ASDA 1992; Rechstchaffen and Kales 1968) by a unique trained scorer (IA) blind to the experimental condition. The total sleep period was the time elapsed from lights off to wake up times, while total sleep time was the cumulated duration of stages 1 to 4 (nonREM sleep) and REM sleep. The wakefulness after sleep onset duration was the duration of wakefulness from sleep onset to wake up time. The arousal index was the number of arousals per sleep hour. Apnea was defined as the cessation of airflow for $10 \mathrm{~s}$ or more, and hypopnea as a $50 \%$ reduction in airflow followed by arousal. The apnea-hypopnea index was the number of apnea

Table 1. Schedule for Sleep and Challenge Sessions

\begin{tabular}{ll}
\hline Time & \\
\hline Session 1 & Event \\
Day 1 & Low-tryptophan diet at home \\
Day 2 & Low-tryptophan diet at home \\
Day 3 & Arrive fasting at clinical investigation center \\
7:30 AM & Plasma tryptophan drawn \\
7:45 AM & Voiding urine \\
8:50 AM & Mood ratings (Hamilton, POMS) \\
9:00 AM & Onset of daytime urine collection \\
& Administer either tryptophan-free amino-acid or placebo in double-blind fashion \\
10:30 AM & Staying quiet, reading or watching TV \\
10:30 AM-3:30 PM & Plasma tryptophan drawn, mood ratings \\
3:30 PM & Eating low-tryptophan snacks \\
5:30 PM & Eating low-tryptophan snacks \\
7:30 PM & Onset of night-time urine collection \\
9:00 PM & Plasma tryptophan drawn \\
9:30 PM & Sleep EEG hook-up \\
10:00 PM & Sleep EEG recording \\
11:00 PM-7:30 AM & \\
Day 4 & End of night-time urine collection \\
9:00 AM & Tryptophan-rich breakfast \\
9:30 AM & Repeat procedure from session 1 (2 ${ }^{\text {nd }}$ RTD/placebo drink administered at least 7 days from session 1) \\
Session 2 &
\end{tabular}


and hypopnea per sleep hour. REM sleep latency was the interval between first epoch of stage 2 and the first epoch of REM sleep. REM sleep latency corrected was calculated by subtracting the intervening wakefulness from the REM latency. The number and the duration of each individual REM burst within REM sleep were measured. REM index was the ratio of the number of REM bursts to the REM sleep duration. REM density was the percentage of REM sleep with eye movements (i.e. the cumulated duration of each REM burst divided by the duration of each REM sleep period).

\section{Biochemical Measures}

Venous blood collected at 7:45 A.M. (baseline), 3:30 P.M. (afternoon) and 9:30 P.M. (pre-sleep) was centrifuged after a 2-h interval and the plasma was stored at $-80^{\circ} \mathrm{C}$. Free tryptophan levels (Lucca et al. 1992) were quantified in plasma aliquots at the end of the study by high performance liquid chromotography with colorimetric detection. Total urine was collected from 9 A.M. to 9 P.M. (daytime urine) and from 9 P.M. to 9 A.M. (nighttime urine) in order to obtain daytime and nighttime 6-sulfatoxymelatonin (6-SM) concentration. The 12-h urine volume was measured, and samples stored at $-80^{\circ} \mathrm{C}$. We used an assay kit from Stockgrand Ltd (GB) to measure urinary 6-SM levels. The RIA method was modified from that of Arendt et al. (1985). The assay was performed blind. The intra- and inter-assay coefficients of variation were $6 \%$ and $9.9 \%$ respectively, for $27 \mathrm{ng} / \mathrm{ml}(\mathrm{n}=10)$.

\section{Statistical Analysis}

Tryptophan concentrations, 6-sulfatoxymelatonin excretion and mood ratings were analyzed with a re- peated measure analysis of variance, to evaluate condition (RTD and placebo) and time (morning/afternoon or daytime/nighttime) interactions. Sleep characteristics were compared using a repeated measure analysis of variance, to evaluate condition (RTD/placebo) and order (first/second night) effects. Results were considered to be statistically significant for $p \leqslant .05$.

\section{RESULTS}

\section{Biochemical Analysis}

Tryptophan. The tryptophan-free amino acid mixture caused a mean $77 \%$ decrease (from $10.0 \pm 1.6$ at 7:45 A.M. to $2.4 \pm 0.88 \mu \mathrm{g} / \mathrm{ml}$ at 3:30 P.M., mean \pm SEM, $p<$ $.0001)$ in plasma free tryptophan concentrations $5 \mathrm{~h}$ after ingestion. Then it increased to a mean $5.9 \pm 1.4 \mu \mathrm{g} /$ $\mathrm{ml}$ at 9:30 P.M. (11 $\mathrm{h}$ after the mixture ingestion), a concentration still $41 \%$ of baseline plasma free tryptophan concentration $(p<.05)$. The plasma total tryptophan concentration was $49.1 \pm 1.9 \mu \mathrm{M} / \mathrm{ml}$ at 7:45 A.M. and $13.0 \pm 2.2 \mu \mathrm{M} / \mathrm{ml}$ at 3:30 P.M. (a mean 73\% decrease, $p<$ $.0001)$.

The placebo mixture caused no significant changes in plasma free tryptophan levels $(10.1 \pm 1.2$ at 7:45 A.M., $8.5 \pm 1.2$ at 3:30 P.M. and $8.4 \pm 1.0 \mu \mathrm{g} / \mathrm{ml}$ at 9:30 P.M.) and in plasma total tryptophan levels $(49.0 \pm 2.0 \mu \mathrm{M} /$ $\mathrm{ml}$ at 7:45 A.M., $40.0 \pm 2.5 \mu \mathrm{M} / \mathrm{ml}$ at 3:30 P.M.). There were highly significant condition (tryptophan depletion vs. placebo) $(\mathrm{F}=48, p<.0001)$ and time $(\mathrm{F}=139, p<$ .0001) effects (Figure 1).

Melatonin Metabolite. While 6-sulfatoxymelatonin excretion levels were significantly increased during nighttime compared with daytime $(p<.0001)$, the tryp-

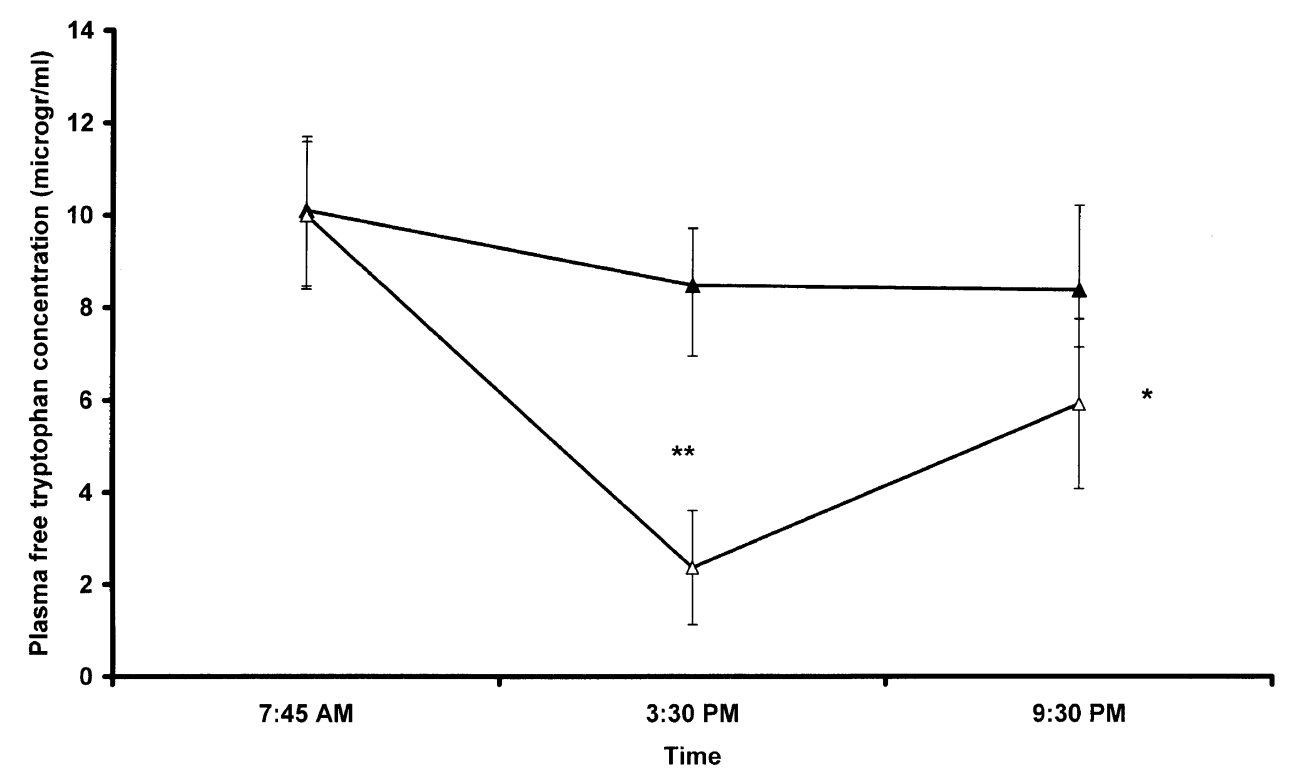

Figure 1. Mean plasma free tryptophan concentrations $(\mu \mathrm{g} / \mathrm{ml})$ following $\operatorname{RTD}(\triangle)$ and placebo $(\boldsymbol{\Delta})$ ingestion. Tryptophan-free amino acid mixture or placebo was given at 10:30 A.M. Results are expressed as mean \pm SEM ( $\mathrm{n}=$ 17). Differences between placebo and RTD conditions were significant at 3:30 P.M. ( $p<$ $.0001)$ and 9:30 P.M. $(p<.05)$. 
tophan-free mixture caused no change in 6-sulfatoxymelatonin excretion $(p=.38)$. During placebo, 6-sulfatoxymelatonin excretion levels were $5.2 \pm 1 \mu \mathrm{g}$ during daytime and $20.2 \pm 3.3 \mu \mathrm{g}$ during nighttime. During RTD, 6-sulfatoxymelatonin excretion levels were $5.9 \pm 0.8 \mu \mathrm{g}$ during daytime and $16 \pm 1.2 \mu \mathrm{g}$ during nighttime.

\section{Psychiatric Scales}

The subjects were normothymic at baseline, with mean mood scores of $1.11 \pm 1.79$ during the placebo session and of $1.11 \pm 2.3$ during RTD. At 3 P.M., mood scores were similar (1.37 \pm 2.19 during the placebo session and $1.74 \pm 1.73$ during RTD). The tryptophan-free mixture had no significant effect or trend on the six POMS subscales. POMS-III score was slightly but significantly ( $p=$ .02 ) decreased in the afternoon, whether the subjects were on placebo (27.5 \pm 1.2 at 9 A.M. and $25.6 \pm 1.5$ at 3:30 P.M.) or on RTD (27.4 \pm 1.3 at 9 A.M. and $22.9 \pm 1.7$ at 3:30 P.M.). The same slight afternoon decrease was observed in POMS-VI score $(p=.03)$, whether the subjects were on placebo (60.0 \pm 1.2 at 9 A.M. and $57.7 \pm 1.1$ at 3:30 P.M.) or on RTD (59.7 \pm 1.5 at 9 A.M. and $55.5 \pm 1.9$ at 3:30 P.M.).

Sleep Measures. There was no significant "order" effect (or "first night effect") and no gender effect for any of the sleep measures.

Sleep Architecture. Sleep latency, total sleep time, total sleep period, duration of wakefulness after sleep onset, stages 1-2 and 3-4 of nonREM sleep, and REM sleep were similar during the placebo session and during RTD (Table 1).

Sleep Fragmentation. The arousal index increased from $4.2 \pm 0.4$ per hour during the placebo period to $6.3 \pm$ 0.9 during RTD $(p<.05)$, with a mean individual increase of $58 \%$. No periodic leg movements were observed. Apnea and hypopnea were absent or extremely rare in all subjects except in one who had a moderate non-symptomatic obstructive apnea-hypopnea syndrome during both the placebo period (apnea-hypopnea index: 26.2/hr) and RTD (apnea-hypopnea index: 23.6/hr). Minimal oxyhemoglobin saturation was within $81-98 \%$ after placebo ingestion and $81-96 \%$ after RTD ingestion, and similar during both periods in all subjects.

First Sleep Cycle. REM sleep latency was significantly longer during RTD than during the placebo period with a mean intra-subject difference of 26 min for REM sleep latency ( $p=.007)$, and $21 \mathrm{~min}$ for corrected REM sleep latency ( $p=.004$; Table 2 and Figure 2). There was a definite increase in REM sleep latency (from +13 to $+75 \mathrm{~min}$ ) in five women and five men, while REM sleep latency was almost unchanged (from -11 to $+2 \mathrm{~min}$ ) in the seven other subjects. The increase was secondary to an increase in the duration of sleep stages 1-2 (Table 3). The first sleep cycle contained $29 \pm 4$ min of stages $1-2$ of nonREM sleep during placebo period versus $49 \pm 9$ min during RTD $(p<.01)$. The duration of slow wave sleep and REM sleep was unchanged during this cycle, but REM density was significantly increased $(p<.05)$ during RTD $(12.3 \pm 2.1 \%)$ compared with placebo period $(8.5 \pm 1.5 \%)$. The mean individual change was a doubling of REM density. REM index tended to increase during RTD $(1.52 \pm 0.16)$ compared with placebo period $(1.27 \pm 0.13)$, but the difference was not statistically significant. During subsequent cycles, REM sleep density increased to the same extent during the placebo period and during RTD (Figure 3). The duration of the second and third sleep cycles was similar during both sessions.

\section{DISCUSSION}

Rapid daytime tryptophan depletion in healthy volunteers significantly decreased plasma tryptophan levels during the day and the evening, followed by alterations in the characteristics of night-time sleep, including increased sleep fragmentation, REM sleep latency, and REM density during first sleep episode.

This is the first study of the effects of daytime RTD on nighttime sleep in healthy subjects. The results differ in some respects from those of Voderholzer et al. (1998)

Table 2. Effect of Daytime Rapid Tryptophan Depletion on Sleep Measures

\begin{tabular}{lcc}
\hline Sleep measures & Placebo & RTD \\
\hline Total sleep period $(\mathrm{min})$ & $453 \pm 8$ & $457 \pm 7$ \\
& $(386-522)$ & $(393-505)$ \\
Total sleep time (min) & $426 \pm 12$ & $425 \pm 10$ \\
& $(312-510)$ & $(342-492)$ \\
Wakefulness after sleep onset (min) & $27 \pm 10$ & $31 \pm 7$ \\
& $(0-128)$ & $(1-83)$ \\
Latency (min) & $16 \pm 3$ & $12 \pm 7$ \\
Stage 2 & $(4-43)$ & $(1-31)$ \\
& $97 \pm 6$ & $123 \pm 9^{* *}$ \\
REM sleep & $(69-150)$ & $(70-184)$ \\
& & \\
Sleep duration (min) & $228 \pm 12$ & $234 \pm 12$ \\
Stages 1-2 & $(141-367)$ & $(151-310)$ \\
& $96 \pm 9$ & $96 \pm 10$ \\
Stages 3-4 & $(15-157)$ & $(22-160)$ \\
& $104 \pm 7$ & $95 \pm 6$ \\
REM sleep & $(41-158)$ & $(44-143)$ \\
& $4.2 \pm 0.4$ & $6.3 \pm 0.9^{*}$ \\
Arousal index (events/h) & $(1.4-7.5)$ & $(2-18)$ \\
&
\end{tabular}

Values are mean \pm SEM; the range is indicated in italics; ${ }^{*} p<.05$, $* * p=.007$. 


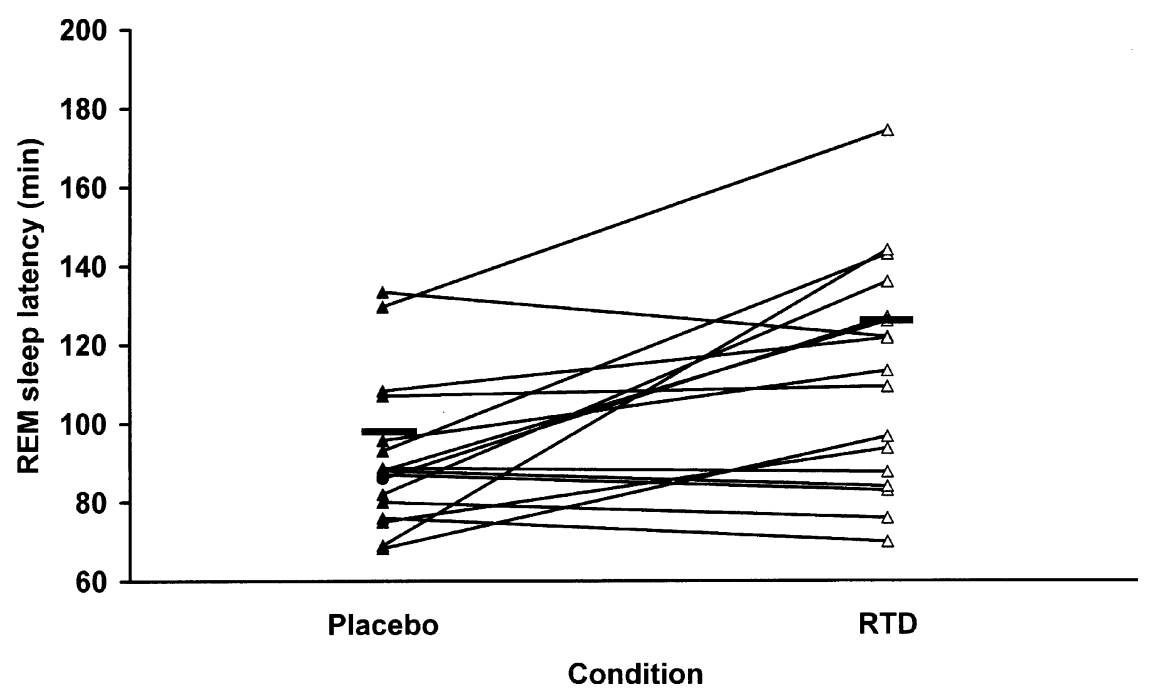

Figure 2. Individual corrected REM sleep latency ( $\mathrm{min}$ ) following $\operatorname{RTD}(\triangle)$ and placebo $(\boldsymbol{\Delta})$ ingestion. A short thick line indicates the mean of the group. REM sleep latency was significantly increased $(p<.004)$ after RTD ingestion.

and Bhatti et al. (1998), who induced RTD during the evening in twelve and eleven healthy subjects. Voderholzer et al. (1998) reported a decrease in stage 2 of sleep, an increase in wakefulness and in the number of REM. Bhatti et al. (1998) reported a decrease in REM sleep latency, REM time and REM $\%$. The discrepancies between these studies and our study possibly result from differences in: (1) timing of nadir tryptophan levels (3 P.M. in our study vs. 11 P.M. in their studies); (2) duration of depletion (13 h vs. $5 \mathrm{~h}$ ) before sleep; (3) content of the placebo mixture (no tryptophan and no amino acid vs. $2.3 \mathrm{~g}$ tryptophan or $25 \%$ RTD); (4) habituation to sleep laboratory (12 h vs. $72 \mathrm{~h}$ ); and (5) inclusion of female subjects. The 5:30 P.M. and 7:30 P.M. low tryptophan snacks, served as meal, could be responsible for the partial recovery of tryptophan levels after eating the snacks. The tryptophan may also be produced from muscle destruction after a prolonged depletion. This would potentially blunt brain serotonin deficiency. However, since $80 \%$ tryptophan depletion results in a 9-40 times reduction in brain serotonin turn over (Nishizawa et al. 1997), 50\% tryptophan depletion should also induce significantly low brain serotonin turn-over. Since the serotonin metabolite 5-hydoxyindoleacetic acid continues to decrease in cerebrospinal fluid $14 \mathrm{~h}$ after RTD in healthy volunteers (Williams et al. 1999), serotonin turnover was probably still low when REM sleep occurred in our subjects. An alternative explanation for the extended REM sleep latency could be that tryptophan levels, although they were still low at 9:00 P.M., were rising at the time of sleep onset. One may imagine that the serotonin-depleted neurons are eager for tryptophan, and would be able to selectively uptake even some small amounts of tryptophan. The newly synthesized serotonin would be released upon upregulated serotonin receptors, resulting in a rebound of serotonergic transmission and a subsequent increased REM sleep latency. Finally, since the rate of brain serotonin synthesis has been shown to be $50 \%$ lower in women than in men at baseline, and

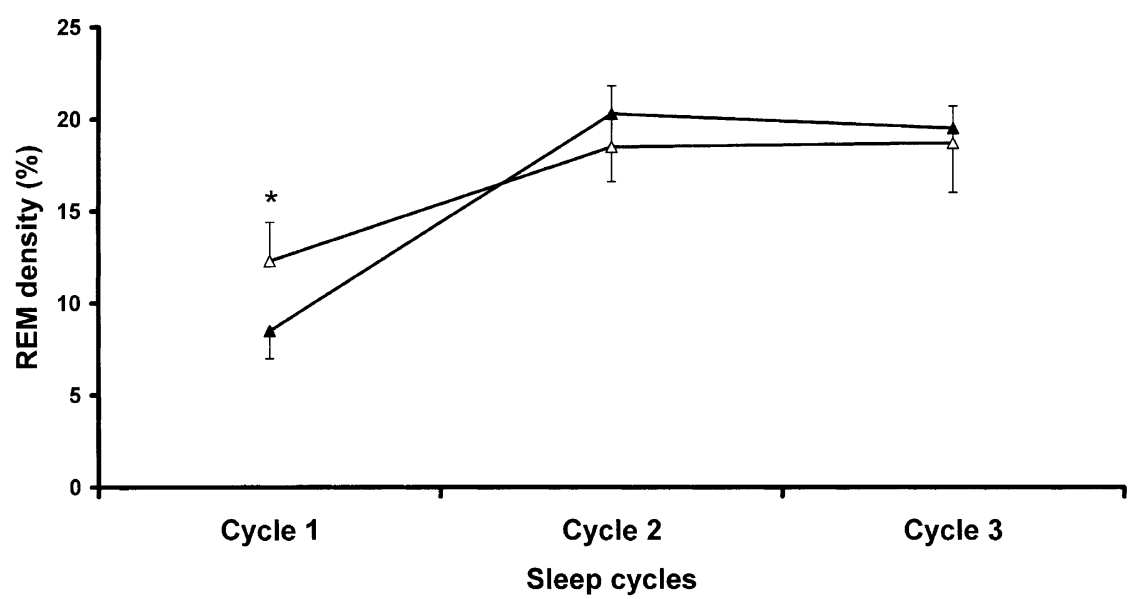

Figure 3. Density of rapid eye movements (percentage of REM sleep) within the first, second and third REM sleep periods following $\operatorname{RTD}(\triangle)$ and placebo $(\boldsymbol{\Delta})$ ingestion. Results are expressed as mean $\pm \operatorname{SEM}(\mathrm{n}=17) .{ }^{*} p<$ .05 . 
Table 3. Effect of Daytime Rapid Tryptophan Depletion on the First Sleep Cycle

\begin{tabular}{lcc}
\hline $\begin{array}{l}\text { Sleep measures } \\
\text { (first sleep cycle) }\end{array}$ & Placebo & RTD \\
\hline Stage 1-2 duration (min) & $29.2 \pm 3.8$ & $49.4 \pm 8.6$ \\
& $(10.7-61.7)$ & $(19.0-123.0)$ \\
Stage 3-4 duration (min) & $43.4 \pm 4.0$ & $46.5 \pm 5.0$ \\
& $(12.3-64.3)$ & $(25.0-84.3)$ \\
REM sleep duration (min) & $13.4 \pm 1.9$ & $12.8 \pm 2.2$ \\
& $(5.0-28.7)$ & $(1.3-33.3)$ \\
REM sleep latency & $96.9 \pm 6.1$ & $123.3 \pm 9.0^{* *}$ \\
(raw, min) & $(69-150)$ & $(70-184)$ \\
REM sleep latency & $91.5 \pm 4.5$ & $112.2 \pm 6.9^{* *}$ \\
(corrected, min) & $(68-133)$ & $(70-174)$ \\
REM index (N/min of & $0.42 \pm 0.04$ & $0.51 \pm 0.05$ \\
REM sleep) & $(0.12-0.68)$ & $(0.14-0.90)$ \\
REM density (\% REM sleep & $8.5 \pm 1.5$ & $12.3 \pm 2.2^{*}$ \\
with REM) & $(1.0-21.3)$ & $(1.3-35.9)$ \\
\hline
\end{tabular}

Values are mean $\pm \operatorname{SEM}\left(\right.$ range); ${ }^{*} p<.05,{ }^{* *} p<.00$

further 90\% lower after RTD (Nishizawa et al. 1997), one may expect sleep to be more disturbed in women than in men at baseline and after RTD. However, we did not find any gender effects on the sleep outcome suggestive of an enhanced female gender susceptibility to RTD. This result parallels the absence of gender difference in REM sleep latency observed in a large group of depressed patients (Reynolds et al. 1990).

High REM density of the first REM sleep period has been early described in depressed subjects (Kupfer 1976). Since RTD did not affect mood ratings in our subjects, this abnormality induced here by RTD is likely not to reflect a depressive mood. The index of REM bursts was unchanged after RTD ingestion, while REM density was doubled, indicating that the observed increase in REM density was secondary to an increase in the duration of REM rather than in their number. This indicates that serotonin might limit the duration of REM rather than the frequency of REM bursts. In depressive patients, the increase in REM density is commonly associated with a decrease in REM sleep latency (Kupfer 1976). This effect has been induced in depressed patients during remission (Moore et al. 1998), and in healthy subjects (Bhatti et al. 1998) by RTD experimentally induced in the afternoon. In our study, a significant increase, not a decrease, in REM sleep latency was found in the normal controls. The reason for this is not clear. The first episode of REM sleep, but not the following episodes, was delayed. REM sleep latency is dependent on circadian rhythms, the shortest REM sleep latency being observed when body temperature is at its lowest (Czeisler et al. 1980). Although we did not monitor body temperature in the subjects, and do not know whether the nocturnal nadir was delayed, we did measure the major urinary melatonin metabolite, which normally varies with a circadian rhythm. Although melatonin derives from serotonin, we did not observe any decrease in diurnal and nocturnal excretions of 6-sulfatoxymelatonin in either subjects on placebo or after RTD, in contrast to a previous report (Zimmerman et al. 1993). However, the amino acid mixture was ingested in the afternoon during this last experiment. This suggests that the effects of RTD on REM sleep latency or on melatonin rhythm may depend on the time elapsed after RTD induction. However, one may keep in mind that a measure of the12-h excretion of melatonin metabolite can detect a change in the amplitude of the melatonin rhythm, but not a delay in the phase of the rhythm. An hourly measure of blood or salivary melatonin could determine the phase changes in melatonin rhythm, but this sampling would disturb sleep. Yet we cannot determine if the delay in REM sleep latency was secondary to a melatonin or temperature phase delay. The effects on REM sleep latency and body temperature of RTD induced at different times of the day would help understand the effect of serotonin on sleep.

Sleep fragmentation indexes increased $50 \%$ in subjects after RTD. However, individual baseline and RTD arousal indexes were within the ranges expected of young healthy subjects. We observed no respiratory events or periodic leg movements, which are common causes of sleep fragmentation. Since we did not record esophageal pressure in the subjects, an increase in upper airway resistance as a cause of arousal cannot be eliminated. Indeed, serotonergic innervation of pharyngeal dilator muscles has been described in animal models (Rose et al. 1995). Although RTD might be expected to facilitate upper airway collapse, this was not the case, however. Only one volunteer presented a mild nonsymptomatic sleep apnea syndrome that was similar following placebo treatment or RTD. Finally, increased sleep fragmentation is observed in insomniacs and patients suffering from depression and could also result from serotonin deficiency. L-tryptophan was widely used as a natural sedative in the 1970s (Wyatt et al. 1970). Thus, one may expect tryptophan depletion to impair sleep continuity. However, RTD did not reproduce in humans the drastic insomnia observed after inhibition of serotonin synthesis by parachlorophenylalanine in cat. Tryptophan depletion and inhibition of serotonin synthesis may therefore alter serotonindependent functions through different mechanisms.

In conclusion, midmorning RTD in healthy subjects has different effects on following REM sleep than evening or afternoon RTD. This suggests that serotonergic control of REM sleep latency, unlike phasic REM events and arousals, may be time-dependent, and may be associated with a circadian rhythm that needs to be identified. Studies dealing with time-dependent effects of RTD on sleep architecture might answer this question. Alternatively, the REM sleep latency may be con- 
trolled by the rate of serotonin synthesis rather than the serotonin level itself.

\section{ACKNOWLEDGMENTS}

The study was founded by Assistance-Publique-Hopitaux-deParis, (Délégation à la Recherche Clinique), grant PHRC AOA94057 and EMUL 930902. We are indebted to Geneviève Barret (CIC) for sleep recordings, to Odile Maugenest, and to Anne-Marie César d'Ornano (Nutrition Department) for preparing the low-tryptophan diets.

\section{REFERENCES}

ASDA (1992): EEG arousals: Scoring rules and examples. A preliminary report from the Sleep Disorders Atlas Task Force of the American Sleep Disorders Association. Sleep 15:174-184

Arendt J, Bojkowski C, Franey C, Wright J, Marks V (1985): Immunoassay of 6-hydroxymelatonin sulfate in human plasma and urine: abolition of the urinary 24-hour rhythm with atenolol. J Clin Endocrinol Metab 60:11661173

Arnulf I, Bejjani BP, Garma L, Bonnet AM, Damier P, Pidoux B, Dormont D, Cornu P, Derenne JP, Agid Y (2000): Effect of low and high frequency thalamic stimulation on sleep in patients with Parkinson's disease and essential tremor. J Sleep Res 9:55-62

Belongia EA, Hedberg CW, Gleich GJ, White KE, Mayeno AN, Loegering DA, Dunnette SL, Pirie PL, MacDonald KL, Osterholm MT (1990): An investigation of the cause of the eosinophilia-myalgia syndrome associated with tryptophan use. N Engl J Med 323:357-365

Benkelfat C, Ellenbogen MA, Dean P, Palmour RM, Young SN (1994): Mood-lowering effect of tryptophan depletion. Enhanced susceptibility in young men at genetic risk for major affective disorders. Arch Gen Psychiatry 51:687-697

Bhatti T, Gillin JC, Seifritz E, Moore P, Clark C, Golshan S, Stahl S, Rapaport M, Kelsoe J (1998): Effects of a tryptophan-free amino acid drink challenge on normal human sleep electroencephalogram and mood. Biol Psychiatry 43:52-59

Cespuglio R, Faradji H, Jouvet M (1983): Voltammetric detection of extracellular 5-hydroxyindole compounds at the level of cell bodies and the terminals of the raphe system: variations during the wake-sleep cycle in the rat in chronic experiments. C R Seances Acad Sci III 296:611-616

Czeisler CA, Zimmerman JC, Ronda JM, Moore-Ede MC, Weitzman ED (1980): Timing of REM sleep is coupled to the circadian rhythm of temperature. Sleep 2:329-346

Delgado P, Charney D, Price L, Aghajanian G, Landis H, Heninger G (1990): Serotonin function and the mechanism of antidepressant action. Reversal of antidepressant-induced remission by rapid depletion of plasma tryptophan. Arch Gen Psychiatry 47:411-413

Fernstrom JD, Wurtman RJ (1974): Brain serotonin content: physiological dependence on plasma tryptophan levels. Science 173:149-152

Giles D, Kupfer D, Rush A, Roffwarg H (1998): Controlled comparison of electrophysiological sleep in families of probands with unipolar depression. Am J Psychiatry 155:192-199

Hamilton M (1960): A rating scale for depression. J Neurol Neurosurg Psychiat 23:56-62

Jouvet M (1972): The role of monoamine and acetylcholinecontaining neurons in the regulation of the sleep-waking cycle. Ergeb Physiol 64:166-307

Jouvet M (1984): Neuromediators and hypnogenic factors. Rev Neurol (Paris) 140:389-400

Kupfer DJ (1976): REM latency: a biological marker for primary depressive disease. Biol Psychiatry 11:159-174

Leyton M, Young S, Benkelfat C (1997): Relapse of depression after rapid depletion of tryptophan. Lancet 349:1840-1841

Lorr M, McNair D (1982): Profile of Mood States - Bipolar Form. San Diego, Educational and Industrial Testing Service

Lucca A, Lucini V, Piatti E, Ronchi P, Smeraldi E (1992): Plasma tryptophan levels and plasma tryptophan/ amino acid ratio in patients with mood disorder, patients with obsessive-compulsive disorder and normal subjects. Psychiatry Res 44:85-91

Moore P, Gillin C, Bhatti T, De Modena A, Seifritz E, Clark C, Stahl S, Rapaport M, Kelsoe J (1998): Rapid tryptophan depletion, sleep electroencephalogram, and mood in men with remitted depression on serotonin reuptake inhibitors. Arch Gen Psychiatry 55:534-539

Nishizawa S, Benkelfat C, Young SN, Leyton M, Mzengeza S, de Montigny C, Blier P, Diksic M (1997): Differences between males and females in rates of serotonin synthesis in human brain. Proc Natl Acad Sci USA 94:53085313

Nurnberger JI, Blehar MC, Kaufman CA, York-Cooler C, Simpson SG, Harkavy-Friedman J, Severe JB, Malaspina D, Reich T (1994): Diagnostic interview for genetic studies. Rationale, unique features and training. Arch Gen Psychiatry 5:849-864

Rechstchaffen A, Kales A (1968): A Manual of Standardized Terminology, Techniques and Scoring System for Sleep Stages of Human Subjects. Los Angeles, UCLA Brain Information Service/Brain Research Institute

Reynolds CF 3rd, Kupfer DJ, Thase ME, Frank E, Jarrett DB, Coble PA, Hoch CC, Buysse DJ, Simons AD, Houck PR (1990): Sleep, gender, and depression: an analysis of gender effects on the electroencephalographic sleep of 302 depressed outpatients. Biol Psychiatry 28:673-684

Rose D, Khater-Boidin J, Toussaint P, Duron B (1995): Central effects of 5-HT on respiratory and hypoglossal activities in the adult cat. Respir Physiol 101:59-69

Smith KA, Fairburn CG, Cowen PJ (1997): Relapse of depression after rapid depletion of tryptophan. Lancet 349:915-919

Trivedi M, Rush A, Armitage R, Gullion C, Grannemann B, Orsulak P, Roffwarg H (1999): Effects of fluoxetine on the polysomnogram in outpatients with major depression. Neuropsychopharmacology 20:447-459

Trulson M, Jacobs B (1979): Raphe unit activity in freely 
moving cats: correlation with level of behavioral arousal. Brain Res 163:135-150

Voderholzer U, Hornyak M, Thiel B, Huwig-Poppe C, Kiemen A, König A, Backhaus J, Riemann D, Berger M, Hohagen F (1998): Impact of experimentally induced serotonin deficiency by tryptophan depletion on sleep EEG in healthy subjects. Neuropsychopharmacology 18:112-124

Williams WA, Shoaf SE, Hommer D, Rawlings R, Linnoila M (1999): Effects of acute tryptophan depletion on plasma and cerebrospinal fluid tryptophan and 5-hydroxyindoleacetic acid in normal volunteers. J Neurochem 72: 1641-1647
Wyatt RJ, Engelman K, Kupfer DJ, Fram DH, Sjoerdsma A, Snyder F (1970): Effects of L-tryptophan (a natural sedative) on human sleep. Lancet 2:842-846

Young SN, Smith SE, Pihl RO, Ervin FR (1985): Tryptophan depletion causes a rapid lowering of mood in normal males. Psychopharmacology (Berl) 87:173-177

Zimmerman RC, McDougle CJ, Schumacher M, Olcese J, Heninger GR, Price LH (1993): Urinary 6-hydroxymelatonin sulfate as a measure of melatonin secretion during acute tryptophan depletion. Psychoneuroendocrinology 18:567-578 